\title{
Treatment of knee prosthesis infections: evaluation of 15 patients over a 5-year period
}

\author{
Katrin Kösters • Reinout van Crevel • \\ Patrick D. J. Sturm • B. Willem Schreurs • \\ Maarten C. de Waal Malefijt • Albert van Kampen • \\ Bart Jan Kullberg
}

Received: 24 May 2008/Revised: 30 June 2008 /Accepted: 1 July 2008/Published online: 15 August 2008

(C) The Author(s) 2008

\begin{abstract}
Our objective was to evaluate different treatment alternatives for total knee arthroplasty (TKA) infection and to compare outcomes depending on adherence to a current treatment algorithm. All patients treated for a first episode of TKA infection between January 2000 and July 2005 were included. Patient records were reviewed and data were extracted retrospectively. Fifteen patients were followed up for a median of 25 months. The cure rate in patients with two-stage exchange of knee prosthesis was higher than in patients who had débridement without implant removal (100 vs $37 \%, p=0.03)$. Cure rates were not different between these two surgical approaches in ten patients who
\end{abstract}

K. Kösters $\cdot$ R. van Crevel $\cdot$ B. J. Kullberg

Department of Internal Medicine,

Radboud University Nijmegen Medical Center,

Nijmegen, The Netherlands

P. D. J. Sturm

Department of Medical Microbiology,

Radboud University Nijmegen Medical Center,

Nijmegen, The Netherlands

B. Willem Schreurs · M. C. de Waal Malefijt • A. van Kampen

Department of Orthopedic Surgery,

Radboud University Nijmegen Medical Center,

Nijmegen, The Netherlands

K. Kösters • R. van Crevel • P. D. J. Sturm • B. Willem Schreurs • M. C. de Waal Malefijt · A. van Kampen · B. J. Kullberg

Nijmegen University Center for Infectious Diseases,

Nijmegen, The Netherlands

K. Kösters $(\square)$

Department of Laboratory Medicine, HELIOS Klinikum Krefeld,

Lutherplatz 40,

47805 Krefeld, Germany

e-mail: kakoesters@gmail.com were treated according to a current treatment algorithm. Success rates for treatment of TKA infections varied considerably with the treatment strategy chosen. Our results support the use of existing algorithms to select patients who are eligible for débridement with retention of the prosthesis or need two-stage exchange of knee implants.

Résumé L'objectif est d'évaluer les différentes alternatives thérapeutiques concernant les infections de prothèses totales du genou et de mettre au point un algorithme de traitement. Patients et méthode: tous les patients traités pour un premier épisode d'infection de prothèse totale du genou entre janvier 2000 et juillet 2005 ont été inclus. Toutes les données concernant les patients et les dossiers médicaux ont été analysées de façon rétrospective. Résultats: 15 patients ont été suivis pendant une moyenne de 25 mois. Les patients ayant bénéficié d'un traitement en deux temps ont des résultats bien supérieurs à ceux qui ont bénéficié d'une simple mise à plat sans ablation d'implants $(100 \%$ vs. $37 \% ; p=0,03)$. Néanmoins, les taux de guérison n'ont pas été différents chez 10 patients qui ont été traités selon un algorithme therapeutique. En conclusion: les taux de succès $\mathrm{du}$ traitement des infections de prothèses totales du genou peuvent varier considérablement selon la stratégie thérapeutique choisie. Nos données suggèrent l'utilisation d' algorithme therapeutique pour sélectionner les patients pouvant bénéficier d'une simple mis à plat sans ablation d'implants, de ceux ayant besoin d'un traitement en deux temps.

\section{Introduction}

Infection of the prosthetic joint after total knee arthroplasty (TKA) occurs in up to $2 \%$ of patients [14]. Various treat- 
ment options exist: débridement with retention of the prosthesis, reimplantation arthroplasty (one- and two-stage procedures), permanent prosthesis removal (resection arthroplasty, arthrodesis), long-term suppressive antibiotic therapy and amputation, of which the least invasive procedure leading to eradication of the infection is preferred. Success rates for the different procedures vary tremendously depending on patient selection, microbiology or duration of follow-up $[2,5,6]$. The choice of a specific surgical treatment strategy often depends on personal experience and tradition rather than on defined criteria. Several treatment algorithms for prosthetic joint infections have been developed in recent years [11-14]. Although not evaluated prospectively, these algorithms recommend choosing a surgical procedure according to predefined criteria, such as duration of symptoms of infection, classification of infection and infecting microorganism.

We reviewed all cases of TKA infection treated at the university hospital in Nijmegen, Netherlands, from January 2000 to July 2005. Our aim was to evaluate treatment outcomes of different treatment options and identify factors possibly associated with treatment failure, including adherence to current treatment algorithms.

\section{Patients and methods}

The study was performed at the Departments of Orthopedic Surgery and Infectious Diseases at Radboud University Nijmegen Medical Center, a 1000-bed university hospital that serves as a referral centre for a population of approximately 2.5 million in the east of the Netherlands.

The study population consisted of all patients with an established diagnosis of infected total knee prosthesis between January 2000 and July 2005. Patients were retrospectively identified through the patient databases of the Departments of Infectious Diseases and Orthopedic Surgery. Cases had to meet at least one of the following criteria for TKA infection: presence of a sinus tract communicating with the joint, growth of the same microorganism from at least two surgical biopsies, signs of acute inflammation on histopathological examination or purulence of synovial fluid or at the implant site [14].

Clinical signs and symptoms (including pain, decreased function, sinus tracts, temperature, C-reactive protein level, white blood cell count and erythrocyte sedimentation rate), infecting organisms, delay between onset of symptoms and surgical treatment, surgical interventions, antibiotic therapy and comorbidity were recorded. Infections were classified according to onset of symptoms after prosthesis placement into early ( $\leq 3$ months after surgery), delayed (3-24 months after surgery) and late ( $\geq 24$ months after surgery) with acute or chronic onset.

Treatment was termed successful when no clinical, radiological or laboratory findings suggesting ongoing infection or relapse were present at follow-up. Follow-up lasted until July 2006 or until treatment failure was established. Treatment strategies were compared with the treatment algorithm developed by Zimmerli and colleagues (Fig. 1) [13, 14].
Fig. 1 Algorithm for treatment of prosthetic joint infections $[13,14]$

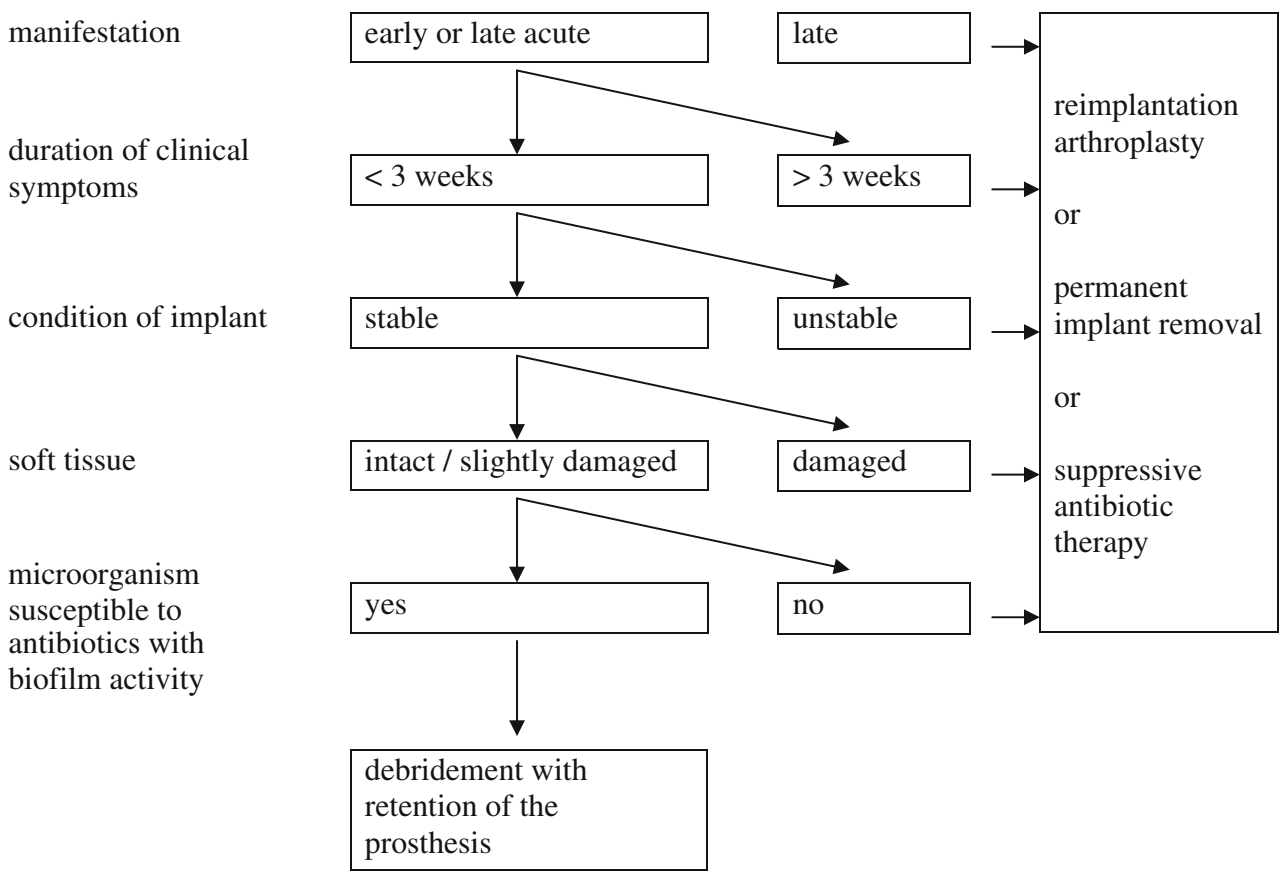


Statistical analysis Non-parametric data were analysed with Fisher's exact test.

\section{Results}

\section{Clinical presentation}

Of 26 patients treated for infected knee prostheses, 15 met the diagnostic criteria for TKA infection. All but five patients met at least two criteria for the diagnosis of infection. The median age at diagnosis of infection was 60 (25-84) years. Seven patients were women. Median followup from surgical intervention was 25 (2-61) months.

The underlying disease that led to total knee arthroplasty was degenerative joint disease in eight patients, rheumatoid arthritis in six patients and haemophilic arthropathy in one patient. Five patients had received immunosuppressive medication [azathioprine, systemic corticosteroids, antitumour necrosis factor (TNF) alpha]. The laboratory characteristics at the time of diagnosis are summarised in Table 1 . Six infections were early infections occurring after a median of 17 (1-63) days after implantation. Two infections were delayed (median: 95 days, range: 91-280) and seven were late infections (median: 1,995 days, range: 728-7,000). Treatment delay, defined as time between onset of first symptoms and surgical intervention, ranged from one day to five years. From 13 of 15 patients, a causative microorganism was isolated. Two episodes were mixed infections, and in two patients no microorganism was identified (Table 1).

\section{Treatment and outcome}

Eight patients were treated with débridement and retention of the prosthesis. One patient was treated with prosthesis removal and arthrodesis, six patients underwent two-stage exchange of the TKA with intermittent placement of a gentamicin spacer and none of the patients received long-term suppressive antibiotic therapy only. The median interval between prosthesis removal and reimplantation was four months (range: 4 5 months). In all cases, cement containing gentamicin was used for the replacement prosthesis.

All patients were treated with long-term antimicrobial therapy, which was adapted after microbiological results were available. Of six patients with two-stage exchange, five received clindamycin monotherapy for a median duration of six (4-6) weeks and one received combination therapy (clindamycin and rifampicin) for six weeks. Of the eight patients who retained their knee prosthesis, five received antibiotic monotherapy $(3 \times$ clindamycin, $1 \times$ amoxicillin and $1 \times$ ciprofloxacin) and three were treated with combination therapy $(2 \times$ ciprofloxacin and rifampicin and $1 \times$ clindamycin and rifampicin). The duration of antibiotic treatment in these patients lasted from six weeks to lifelong. The patient treated with arthrodesis received combination therapy with ciprofloxacin and teicoplanin for six weeks.

Of the 15 patients with TKA infection, $9(60 \%)$ were treated successfully, i. e. they required no further medical or surgical treatment during a median follow-up of 39 (12-61) months (Table 1).

All six patients who underwent a two-stage exchange were cured after this procedure. Of the eight patients who initially underwent débridement with prosthesis retention three were cured. Five needed further treatment $(p=0.03)$ after a median of eight (2-21) months because of uncontrolled infection. Three of these patients underwent a two-stage exchange of the prosthesis to eradicate the infection, whereas in two patients, the infection was controlled by oral administration of suppressive antibiotics. The patient who underwent arthrodesis has remained infected.

All patients who underwent two-stage exchange arthroplasty were treated according to the algorithm for management of prosthetic joint infections published by Zimmerli et al. $[13,14]$. Of the eight patients who were initially treated with débridement, in retrospect only four fulfilled all criteria for retention of the prosthesis as advocated by Zimmerli et al., i. e. early or late acute infection, duration of symptoms less than three weeks, stable implant, intact or only slightly damaged soft tissue and causative microorganism susceptible to antimicrobial agents with activity against surface-adhering bacteria. Three of these four patients who fulfilled the criteria were treated successfully, whereas treatment failed in all four patients not fulfilling the criteria, almost reaching statistical significance $(p=0.07)$ (Table 1$)$. In the small series of 15 patients, we were not able to demonstrate an association between treatment failure and underlying disease, immunosuppression, mono- or polymicrobial infection or type of antibiotic therapy (Table 2).

\section{Discussion}

In this cohort of patients, treatment of TKA infection with a two-stage exchange had a significantly better outcome than débridement with retention of the prosthesis. Other centres have reported similar high failure rates with component retention compared with a two-stage procedure [3, 8]. In a retrospective review of 31 infected total knee prostheses treated with débridement and prosthesis retention, the success rate was only $23 \%$ after a mean follow-up of 8.8 years, whereas Deirmengian et al. achieved a slightly higher success rate of $35 \%$ in 31 patients undergoing the same procedure. In contrast, prosthesis removal with delayed 


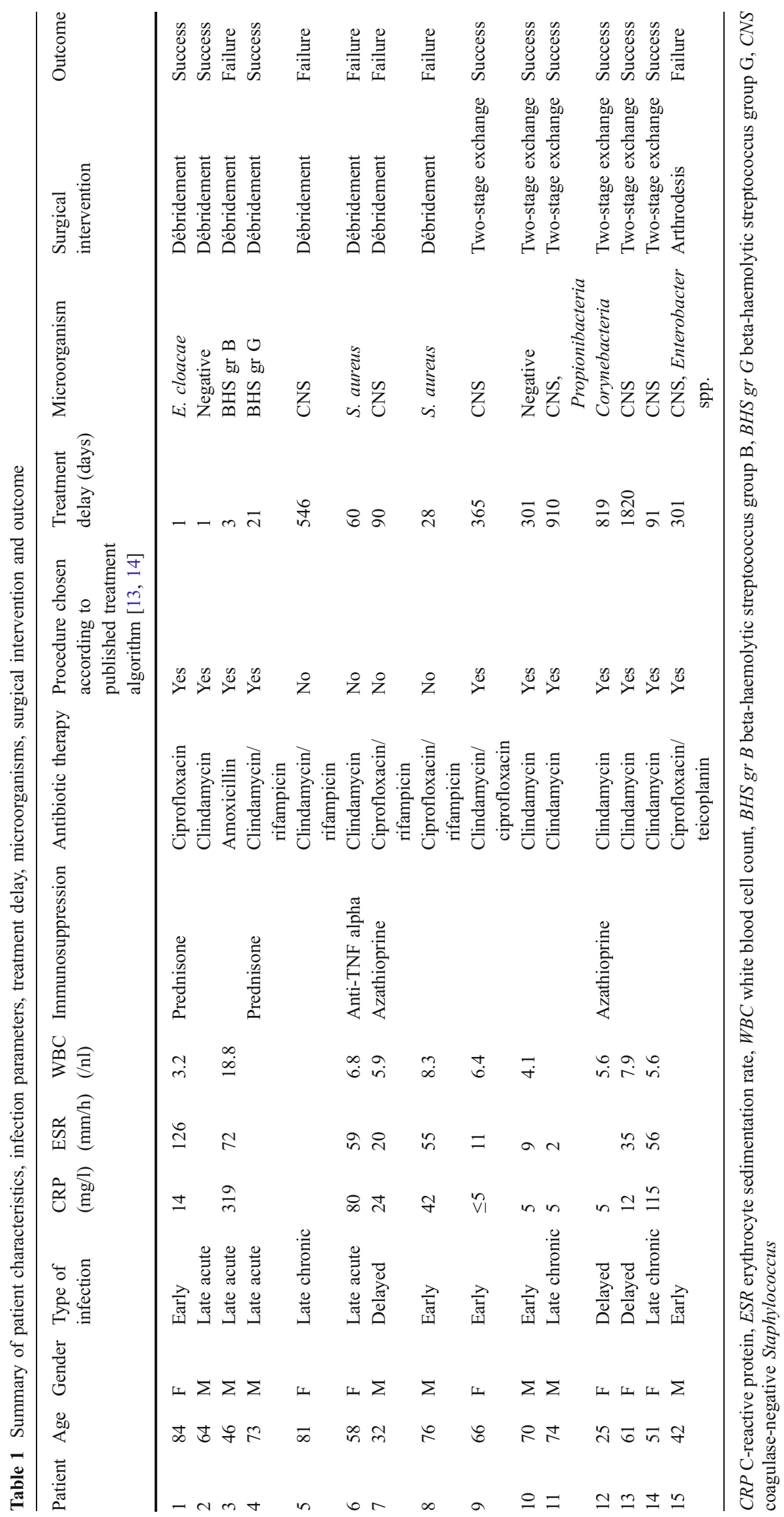


Table 2 Success rate according to underlying disease, immunosuppression, mono- or polymicrobial infection, classification of TKA infection, type of antibiotic therapy and adherence to a published treatment algorithm $[13,14]$

\begin{tabular}{llc}
\hline & & $\begin{array}{c}\text { Success } \\
\text { rate }(n)\end{array}$ \\
\hline Microorganism & Monomicrobial & $6 / 11$ \\
& Polymicrobial & $1 / 2$ \\
Classification & Early & $3 / 5$ \\
& Delayed & $2 / 3$ \\
Antibiotic therapy & Late & $4 / 7$ \\
& Combination including rifampicin & $2 / 4$ \\
& Clindamycin monotherapy & $6 / 8$ \\
Immunosuppression & Other & $1 / 3$ \\
& Yes & $3 / 5$ \\
Underlying disease & No & $6 / 10$ \\
& Degenerative joint disease & $5 / 9$ \\
Treatment & Rheumatoid arthritis & $4 / 6$ \\
& According to protocol & $9 / 11$ \\
& Not according to protocol & $0 / 4$ \\
\hline
\end{tabular}

reimplantation has been reported to be highly successful for eradication of infection. Of 21 patients treated with a twostage exchange and intermittent gentamicin spacer for TKA infection, 19 remained infection free during a mean followup of 24 months [7]. Segawa and colleagues successfully treated 24 of 29 patients with a late chronic TKA infection with delayed exchange arthroplasty, while débridement with retention of the prosthesis resulted in a success rate of only one in 11 [9]. All these studies varied in terms of definition of infection, antimicrobial therapy, types of devices implanted and duration of follow-up.

These findings are in contrast to those reported by other groups: Mont et al. reported a success rate of $71 \%$ for late haematogenous and $100 \%$ for early postoperative TKA infections without removal of the prosthesis [6]. Laffer and colleagues [5] evaluated 35 patients with a total of 40 episodes of TKA infection. They demonstrated that twostage exchange, one-stage exchange and débridement with retention of the prosthesis all had similar success rates, if specific selection criteria for treatment choice were met. Recurrence-free survival was observed in $85 \%$ of patients after two-stage exchange and $95 \%$ after treatment with prosthesis retention. A shorter interval between the onset of infection and the start of treatment was associated with a higher success rate. A similar correlation was described by Barberan et al. [1], with failure rates for conservative treatment of staphylococcal prosthetic joint infection ranging from $17 \%$ in patients with symptom duration of less than one month to $69 \%$ in patients with symptom duration of more than six months. Our study also shows that a shorter duration of symptoms was associated with a more favourable treatment outcome in patients who underwent débridement with prosthesis retention. Treatment delay in three patients treated successfully with retention of the prosthesis was one day to three weeks, whereas in the cases with unsuccessful outcomes the median delay was 8.6 weeks ( 1 day to 1.5 years).

No correlation was found between treatment success and underlying disease, immunosuppression, mono- or polymicrobial infection or type of antibiotic therapy (i.e. inclusion of rifampicin for susceptible microorganisms). This might be due to the small sample, although other groups were also unable to identify any of these factors as predictors of treatment failure $[4,10]$.

Open débridement with prosthesis retention is the least invasive method that can eradicate infection of prosthetic joints, and therefore, an appealing surgical strategy. Although this procedure was not as successful as a delayed exchange procedure in our series as well as in those studies mentioned earlier [3, 8], careful patient selection is likely to yield favourable results comparable with more invasive strategies, as has been suggested by Laffer et al. [5]. This notion was confirmed in our series, where débridement and retention of the prosthesis was successful for three of four patients who did fulfil the suggested criteria. In contrast, infection was not controlled with débridement and retention in all four patients in whom the regimen chosen was not in agreement with the newly proposed algorithm which was published after most of our cases were recruited. In retrospect, these patients did not meet all criteria (early postoperative or acute haematogenous infection, duration of clinical symptoms less than three weeks, stable implant with intact soft tissue and microorganism susceptible to antibiotics with activity against surface-adhering bacteria) subsequently advocated by Zimmerli et al. for treatment with retention of the prosthesis. According to the treatment algorithm a more invasive strategy should have been chosen and might have been more successful for these cases. All patients in our series who received an exchange arthroplasty were treated in keeping with the proposed treatment algorithm $[13,14]$ and showed a successful outcome.

The small sample size of this study did not allow the performance of statistical analysis to identify other risk factors for treatment failure. It also had the inherent limitation of retrospective studies.

In conclusion, good results were obtained with two-stage revision of infected TKA. Outcome of débridement with retention of the prosthesis depended on whether defined selection criteria for this procedure were fulfilled. Our results support the use of an existing treatment algorithm developed by Zimmerli et al. to select the appropriate surgical procedure for patients with TKA infection and underline the importance of a short treatment delay for successful débridement with prosthesis retention. 
Open Access This article is distributed under the terms of the Creative Commons Attribution Noncommercial License which permits any noncommercial use, distribution, and reproduction in any medium, provided the original author(s) and source are credited.

\section{References}

1. Barberan J, Aguilar L, Carroquino G, Gimenez MJ, Sanchez B, Martinez D, Prieto J (2006) Conservative treatment of staphylococcal prosthetic joint infections in elderly patients. Am J Med 119:993.e7-993.e10

2. Burger RR, Basch T, Hopson CN (1991) Implant salvage in infected total knee arthroplasty. Clin Orthop Relat Res 273:105-112

3. Deirmengian C, Greenbaum J, Lotke PA, Booth RE Jr, Lonner JH (2003) Limited success with open debridement and retention of components in the treatment of acute Staphylococcus aureus infections after total knee arthroplasty. J Arthroplasty 18:22-26

4. Hartman MB, Fehring TK, Jordan L, Norton HJ (1991) Periprosthetic knee sepsis. The role of irrigation and debridement. Clin Orthop Relat Res 273:113-118

5. Laffer RR, Graber P, Ochsner PE, Zimmerli W (2006) Outcome of prosthetic knee-associated infection: evaluation of 40 consecutive episodes at a single centre. Clin Microbiol Infect 12:433-439
6. Mont MA, Waldman B, Banerjee C, Pacheco IH, Hungerford DS (1997) Multiple irrigation, debridement, and retention of components in infected total knee arthroplasty. J Arthroplasty 12:426-433

7. Pitto RP, Castelli CC, Ferrari R, Munro J (2005) Pre-formed articulating knee spacer in two-stage revision for the infected total knee arthroplasty. Int Orthop 29:305-308

8. Schoifet SD, Morrey BF (1990) Treatment of infection after total knee arthroplasty by debridement with retention of the components. J Bone Joint Surg Am 72:1383-1390

9. Segawa H, Tsukayama DT, Kyle RF, Becker DA, Gustilo RB (1999) Infection after total knee arthroplasty. J Bone Joint Surg Am 81:1434-1445

10. Tattevin P, Cremieux A, Pottier P, Huten D, Carbon C (1999) Prosthetic joint infection: when can prosthesis salvage be considered? Clin Infect Dis 29:292-295

11. Tsukayama D, Goldberg VM, Kyle R (2003) Diagnosis and management of infection after total knee arthroplasty. J Bone Joint Surg Am 85:75-80

12. Tsumura H, Ikeda S, Ono T, Itonaga I, Taira H, Torisu T (2005) Synovectomy, debridement, and continuous irrigation for infected total knee arthroplasty. Int Orthop 29:113-116

13. Zimmerli W, Ochsner PE (2003) Management of infection associated with prosthetic joints. Infection 31:99-108

14. Zimmerli W, Trampuz A, Ochsner PE (2004) Prosthetic-joint infections. N Engl J Med 351:1645-1654 